\title{
THE INTEGRATED UNIT SIMULATION SYSTEM: QUANTIFYING INDIVIDUAL AND SMALL UNIT MISSION EFFECTIVENESS ON THE BATTLEFIELD OF THE FUTURE
}

\author{
Victor E. Middleton \\ Simulation Technologies, Inc. \\ Suite 748, 111 Building \\ 111 West First St. \\ Dayton Ohio 45402
}

\author{
John O'Keefe IV \\ US Army Natick Research, Engineering, and \\ Development Center \\ ATTN:SATNC-AA \\ Kansas ST \\ Natick MA 01760-5015
}

\begin{abstract}
The US Army Natick Research Development \& Engineering Center is developing the Integrated Unit Simulation System (IUSS) in response to a recognized need for analysis tools to evaluate equipment, operational policy, and training within a system context. The IUSS is based on the philosophy of the Soldier as a System: equipment and other contributors to the soldier's performance considered as a synergistic whole. The IUSS methodologies simulate the Soldier System at multiple levels of interest, starting with the effects of equipment and battlefield stressors (e.g., chemical challenge and the subsequent burdens imposed by individual protection) on a taxonomy of human performance abilities and ultimately translating these effects to unit level measures of performance.
\end{abstract}

\section{INTRODUCTION}

The US Army Natick Research, Development \& Engineering Center (Natick), supported by Simulation Technologies, Inc. (STI) and others such as the Dismounted Warfighting Battle Lab, is currently developing the Integrated Unit Simulation System (IUSS) to provide a comprehensive analysis environment for the evaluation of Soldier Systems' survivability and effectiveness. The core of the IUSS is an architecture which supports multiple-component simulation models and associated tools for assessment of analysis results. IUSS features include:

- An integrated analysis environment

- An open extensible architecture for integration of multiple models and data bases

- Measures of Soldier System performance as a function of small unit operability \& survivability

- A flexible paradigm for integration of equipment effects and battlefield stressors at individual and unit levels

- Multi-platform application utilizing graphical user interface
The goals of this development are to:

- Simulate individual small unit operability and survivability

- Analyze current soldier system performance

- Project future soldier system capabilities

Development of the IUSS has relied heavily on multi-agency/multi-service coordination, with respect to issues ranging from high resolution combat and human performance models to questions of interface with network applications such as Distributed Interactive Simulation (DIS), and higher echelon models such as Janus. The IUSS has concentrated on the integration of currently accepted tools and methodologies, while seeking to provide new capability by making these tools work together. The IUSS provides for the first time a task network simulation with explicit links to both clock-driven events and geographical features, capable of integrating a dynamic data base of the soldier's "world", to describe the functional environment of his mission task processes. The IUSS also provides a detailed, well-defined, structured, and auditable simulation sequence to map the effects of battlefield stressors and Soldier System equipment component constraints, first to the potential capability of the individual soldier, then to measures of task performance for those individuals, and ultimately to unit /mission performance metrics.

\section{THE PROBLEM}

Army programs concerned with the development and fielding of new equipment, for example, the Soldier Integrated Protective Ensemble (SIPE) program and Soldier Modernization Plan Initiatives, must support increasingly complex mission requirements, and allow the soldier to survive increasingly hostile and sophisticated threat environments. Budgets are tight. New equipment must be cost-effective, and it must be capable of a priori demonstration of that fact. The 
individual soldier is already burdened with an excessive load. New equipment must replace existing equipment, reducing the soldier's load while maintaining or increasing mission capabilities. This is the rationale behind the philosophy of the Soldier as a System: equipment and other contributors to the soldier's performance considered as a synergistic whole, rather than as a series of isolated factors.

Today's rapidly changing geo-political climate has highlighted the services' need for rapid response analyses to answer questions of survivability and operability in novel threat environments. The US military has responsibility for operations in multiple theaters, potentially facing a wide variety of unique hazards and adverse environmental conditions. The Research and Development (R\&D) community must have a means of rapidly and realistically assessing potential battlefield hazards and operational capability in the face of that hazard.

The Army today, faces a major challenge to realize the promise of today's (and tomorrow's) technologies for the next generation soldier, in the face of shrinking budgets and evolving missions. The IUSS is designed to help meet this challenge, by providing the means to assess the combat capability of the Soldier System, by supporting Battle Lab training initiatives, and ultimately, by providing the analytical basis for operational decision aids. Perhaps the most important aspect of the IUSS development effort is the definition of a common framework for discussion of Soldier System issues through definition of a standard representation of the Soldier System, one that is shared by everyone from war fighters to $R \& D$ analysts.

At present there are a variety of computer modeling tools designed to assist the military analyst. In general, these models are cumbersome to use, requiring a high degree of operator expertise, significant investment in input data preparation, and skilled analyst interpretation of results. Furthermore, they do not allow the easy addition of novel threats or flexible definition of new weapon systems and/or protection against those weapons. These limitations impede the rapid turnaround of analyses to answer the requirements of current events, and subsequently slow the response of research and development efforts in support of the rapid procurement and fielding of new equipment. The shortcomings of current analytical capability have been formally recognized by the Army Materiel Command as a Mission Area Analysis Deficiency of high priority (\#37, TROSCOM 89-034).

The IUSS is addressing this deficiency by attempting to provide a comprehensive understanding of a unit's (or an individual's) ability to perform a combat mission. This ability must be the ultimate measure of merit for decision makers. The IUSS in acknowledgment of this, is designed to parallel the evolution of the Soldier System concept by combining historically disparate models of different aspects of the soldier and his combat systems into an integrated representation of the battlefield, with measurement of unit mission effectiveness as the goal.

\section{ADDRESSING THE NEEDS OF THE SOLDIER}

The value of analytical tools, such as the IUSS, can ultimately be measured only in terms of their benefits to the soldier. Historically products from the R\&D community have addressed $R \& D$ perceptions of the soldier's need, all to often this perception has been at odds with the war fighters' reality. A key component of the IUSS philosophy is the need to provide realistic simulations of the operational environment, and, additionally, to describe that environment in the same language used by combat soldiers as they fight and train.

The IUSS is intended first and foremost to assist the players in the $R \& D$ arena in focusing on the real needs of the Soldier System. The IUSS has been designed to be accessible to both the developer and the user, to facilitate the exchange of information on projected developments. For example, the IUSS supports the demonstration of proposed equipment or other innovations as virtual prototypes, computer simulations of operational concepts which can be viewed in realistic combat settings. Construction of virtual prototypes is a quick, cheap method for early assessment of concept viability, and additionally provides a strawman to elicit end user feedback. Such a conduit for information will ensure that the R\&D community is more responsive to the operational community, with products more focused on their needs and less time required to field those products.

\section{THE IUSS PROGRAM PLAN}

Since the concept of the Soldier System is not well supported by considering equipment in isolation, the IUSS must support examination of issues associated with training, doctrine, and operational concerns, both in-and-of themselves and as interacting factors. The IUSS is not developing new models to represent these factors, but rather integrating available methodologies according to the framework provided by a structured definition of the Soldier System. This process provides a solid scientific basis for Soldier System studies and analyses, and is a critical element in optimizing the combat capability of the next generation soldier.

IUSS development is following a multi-stage plan:

\section{NEAR TERM}

- Integration of Current Methodologies and Data Bases for Representing the Dismounted Soldier

- Development of Soldier System Performance Baselines 
MID TERM

- Interoperability With DIS and other Soldier Models

- Virtual Prototyping of Soldier System Equipment

FAR TERM

- "One Stop Shop" for Soldier System

- Performance

- Vulnerability

- Soldier System "Engine" for Virtual Reality Simulation with Seamless Reconfiguration for Both

- Soldier

- Simulation

Ultimately, a goal of the IUSS is to help realize the promise of such technologies as virtual reality to support the Soldier as a System. It is essential that the 21 st century soldier reap the full benefits of current and evolving technological advances. Modeling and simulation can contribute more and more to this process with advanced analytical tools applied to the mission of providing the best possible equipment for the soldier, and ensuring that this equipment is used to optimize operational capability.

\section{SIMULATION FEATURES}

The IUSS architecture is designed to support estimation of individual and unit capability for a broad spectrum of applications. Representations of battlefield threats and other stressors are integrated with models of soldier equipment and related factors to estimate soldier performance.

The IUSS focuses on the fundamental relationship between a soldier's psycho-physiological state and the ability to perform discrete mission tasks. Defining module data interfaces in terms of this relationship allows the IUSS to deal with each module in terms of its effects on an underlying data structure - the Soldier System. This facilitates aggregation of effects to unitlevel measures of effectiveness, and allows estimation of mission performance and associated costs.

\section{ANALYZE AS WE FIGHT AND TRAIN}

The IUSS models combat according to the Battlefield Operating Systems Tasks (BOS-T) as defined in the Army Training and Evaluation Program and Mission Training Plans (ARTEP/MTPs), ensuring a common framework for analysis, training, and combat operations, and enhancing communication between all the players. Missions are represented in the IUSS as simulation networks, each of whose nodes represent a BOS task. Shown in Figure 1 is a stylized representation of a set of mission tasks and the corresponding IUSS task network.

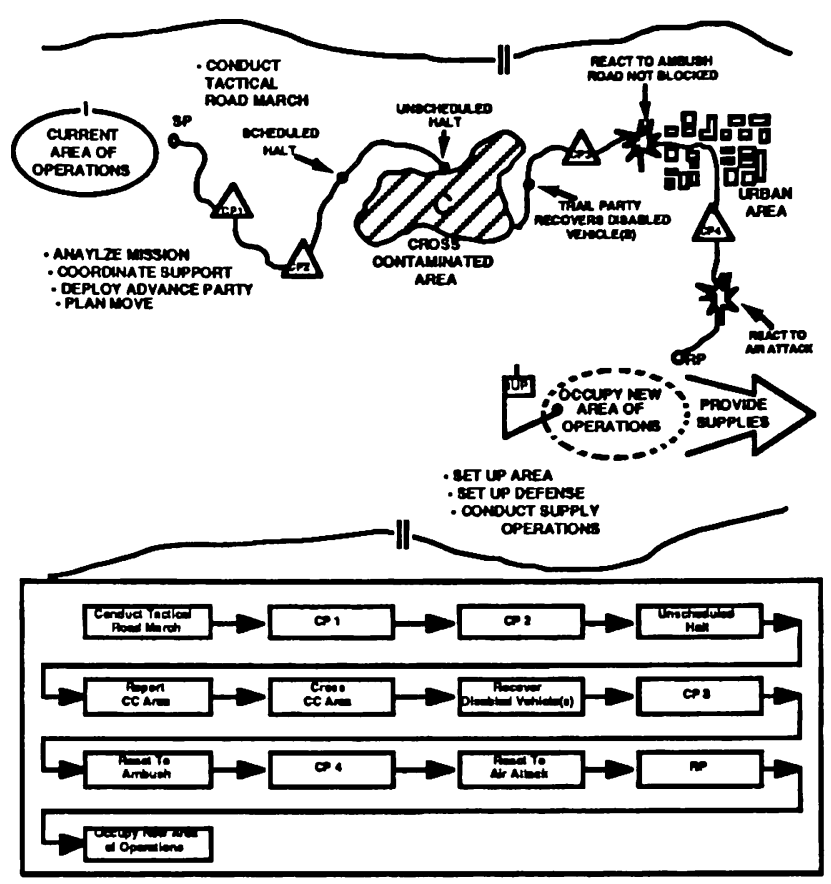

Figure 1 Mission and IUSS Task Networks

For the first time in history, all of the players in the soldier modernization process: combat developers, training developers, and materiel developers will be working off of the same analytical data base. The IUSS, by adopting the form and methods of the ARTEPs, expands the concept of "Train as we fight and fight as we train" to include "Analyze as we fight and train".

Furthermore, the IUSS architecture is not restricted to current BOS-T definitions; it is designed to facilitate the implementation of new processes which can represent new tactics and the employment of novel weapons or equipment. This supports the central concept of the soldier system that equipment cannot be examined distinct from questions of training and operational employment. New systems for the soldier, may modify the way he fights. If this occurs, analysis capability must be able to respond to such modifications rapidly and with minimal modification.

\section{BOS-T SIMULATION LOOP}

As shown in Figure 2, each network task node is basically a simulation loop. The primary node input is the unit resource stream, representing individual soldiers and equipment assigned to the simulated mission tasks. The node loop begins with an evaluation of the assigned unit's ability to perform the given task. The unit may be fully mission capable, in which case the normal simulation process for this task type is initiated. Alternatively, the unit may require some form of reorganization (e.g., reassignment of unit duties to alternate personnel, replenishment of 
unit resources, addition of new personnel) before proceeding with task performance. In the worst case, the unit may be unable to continue, necessitating a task abort and mission failure.

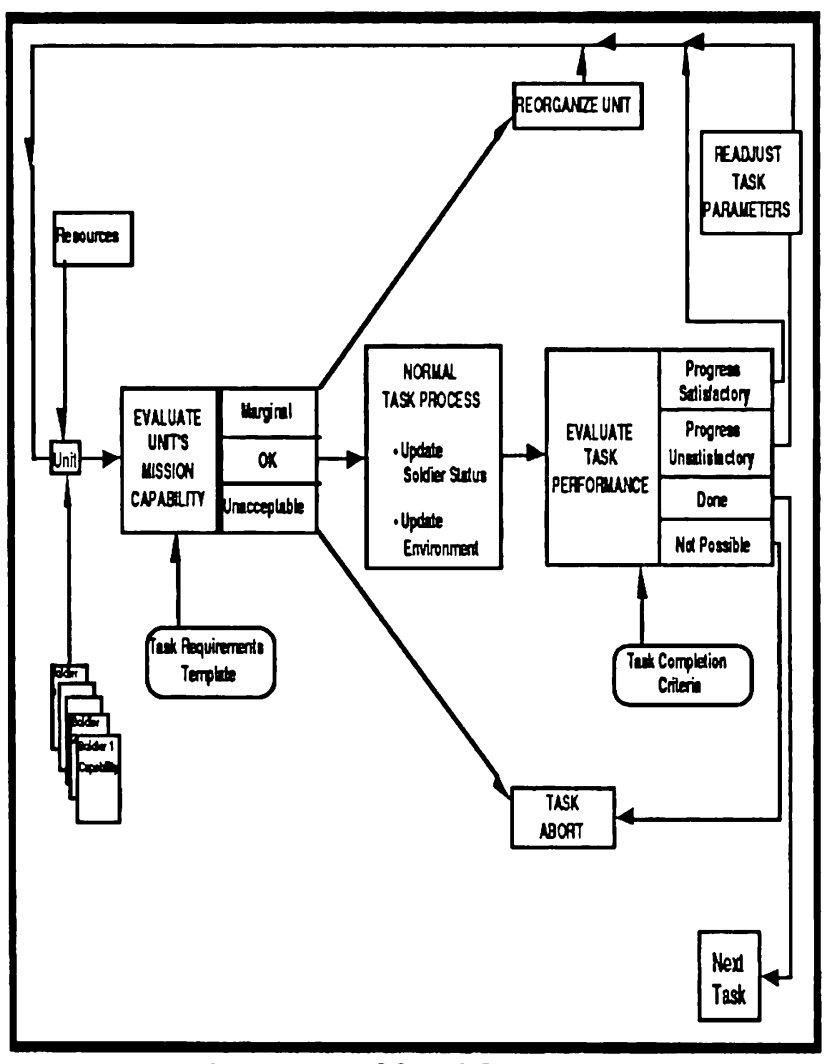

Figure 2 IUSS BOS-T Loops

For each iteration of the loop, evaluation of unit capability results in the assignment of performance parameters (e.g., rate, efficiency) followed by incremental simulation of task processes. At the conclusion of each iteration, the system evaluates the task progress. If the task is complete, the simulation proceeds to the next network task. If the task is incomplete, but progressing normally, the loop for this task node is repeated, evaluating current unit capability (as updated after performance of the simulation process during the last time step) to continue with the task. If the task is not proceeding within defined parameters (e.g., on a Move Tactically task if the directional errors induced by navigational difficulties have drawn the unit off course), some readjustment of task parameters may be required (e.g., the unit commander must calculate a new course). If the task performance is not correctable (e.g., the unit is hopelessly lost), the task is aborted.

\section{TASK PROCESS SIMULATION}

The core of the above network task is the task process simulation, the actual model of the task function, as opposed to the logics which determine process alternatives and functional parameters. The task process simulation implements those phases of the performance methodology which modify the status of the simulated soldiers or the battlefield itself. The process begins by calculating the performance costs of the task, examining the battlefield environment for the stressors affecting the unit's soldiers, updating the status of the soldiers based on the effects of those stressors and the task performance costs, and in turn updating the status of the battlefield in response to the results of task performance.

The task process approach follows the object oriented programming paradigm, allowing simulation of the task as an encapsulated function, a "black box" which can be replaced according to the resolution requirements of a given analysis, and the fidelity of available data to support that process. This also allows the incremental inclusion of the representation of multiple stressors, and the replacement of specific process models as more sophisticated (and hopefully more accurate) models become available.

\section{SUMMARY}

The IUSS was designed to facilitate the application of the $R \& D$ process to the production of improved equipment for the soldier. However, the concept of the Soldier System is not well supported by considering equipment in isolation, and consequently, for the IUSS to achieve its objective, it must also support examination of issues associated with training, doctrine, and operational concerns, both inand-of themselves and as interacting factors.

Coordination of the $R \& D$ process, across the boundaries of materiel, training, and combat developments, requires a common language and a single standard framework for viewing Soldier System issues. The IUSS, by adopting the form and methods of the Army training and evaluation plans, expands the concept of "Train as we fight and fight as we train" to include "Analyze as we fight and train".

The IUSS is resulting in the formation of a structured definition of the Soldier System. This structured definition includes the construction of baselines for Soldier System performance and explicit recognition of the complex relationships between the multiple facets of the modern battlefield. This process provides a solid scientific basis for Soldier System studies and analyses, and is a critical element in optimizing the combat capability of the next generation soldier.

Tomorrow's military will have to meet the diverse challenges of a new, and rapidly changing, geopolitical climate with a smaller force structure, and as a consequence, tomorrow's soldier must be more lethal, mobile, and survivable to achieve mission objectives. It is essential that the 21 st century soldier reap the full benefits of current and evolving 
technological advances, and these must be applied throughout the equipment life cycle, from initial development to fielded use. Modeling and simulation can contribute to this process with advanced analytical tools applied to the mission of providing the best possible equipment for the soldier, and ensuring that this equipment is used to optimize operational capability.

\section{ACKNOWLEDGMENTS}

The work described herein was supported primarily under contract DAAK60-91-C-0125 with the US Army Natick Research Development and Engineering Center. Preliminary findings have been presented at the 61st Annual Military Operations Research Society Symposium. The opinions expressed are solely those of the authors and do not represent any official US Army position.

\section{REFERENCES}

US Army ARTEP 7-8-MTP, Mission Training Plan for the Infantry Rifle Platoon and Squad, dated September 1988).

Vic Middleton, Integrated Unit and Soldier Systems Survivability and Effectiveness Evaluation Roadmap, dated January 1992).

TROSCOM FY89 Mission Are Development Plan, Headquarters US Army Troop Support Command, 1989.

Paul F. Gorman, Supertroop VIA I-Port: Distributed Simulation Technology for Combat Development and Training Development, dated August 1990

\section{AUTHOR BIOGRAPHIES}

VICTOR E. MIDDLETON is a mathematician under contract to Simulation Technologies, Inc. and is responsible for the development of simulation algorithms for the Integrated Unit Simulation System.

JOHN A. O'KEEFE IV a former U.S. Army Major, is a senior operations researcher with the Concepts Analysis Division Advanced Systems Directorate of the US Army Natick Research Development and Engineering Center and is the contract monitor for development of the Integrated Unit Simulation System. 